\title{
Heartbeat: an increase in preventable cardiovascular deaths during the COVID-19 pandemic due to avoidance of medical care
}

doi:10.1136/heartjnl-2020-318759

Catherine M Otto

Patients with cardiovascular disease (CVD) have an increased mortality risk with COVID-19 infection yet several studies have shown fewer hospital-based CVD diagnoses and procedures during the COVID-19 pandemic. In this issue of Heart, Wu and colleagues ${ }^{1}$ show that despite a decrease in the number of patients presenting with an acute CVD event there was an $8 \%$ excess of CVD deaths in England between March and June 2020 (during the COVID-19 pandemic), compared with the previous 6 years (figure 1). About $1 / 2$ of these deaths occurred outside the hospital with the most frequent causes of CVD death being stroke $(35.6 \%)$, acute coronary syndrome (24.5\%), heart failure (23.4\%) pulmonary embolism $(9.3 \%)$ and cardiac arrest $(4.6 \%)$. Most of these deaths were not related to a known COVID-19 infection, suggesting they were most likely due to delays in seeking medical care or undiagnosed COVID-19 infection.

As Singh and $\mathrm{Newby}^{2}$ emphasise in an editorial: 'the evidence presented by Wu and colleagues ${ }^{1}$ provides us with an important message to our patients and society: it is important to seek emergency medical attention for symptoms indicative of serious life-threatening cardiovascular disease even during the height of the pandemic. Here, the risk of fatal stroke and myocardial infarction outweighs the COVID-19 risk to the patient, and the healthcare system had capacity within acute specialities outside of the intensive care and dedicated COVID-19 units to provide life-saving treatments. This ultimately begs the question: is the fear of disease worse than the disease itself?'

Another important study in this issue of heart describes a 12-year cohort study of 419 patients with infective endocarditis in South Korea. ${ }^{3}$ Overall, hospital mortality was $14.6 \%$ with risk factors for mortality including aortic valve infection, Staphylococcus aureus, neurological complications multi-organ failure, and an increased number of comorbidities. Surgical intervention was associated

Correspondence to Professor Catherine M Otto, Division of Cardiology, University of Washington, Seattle,WA 98195, USA; cmotto@uw.edu
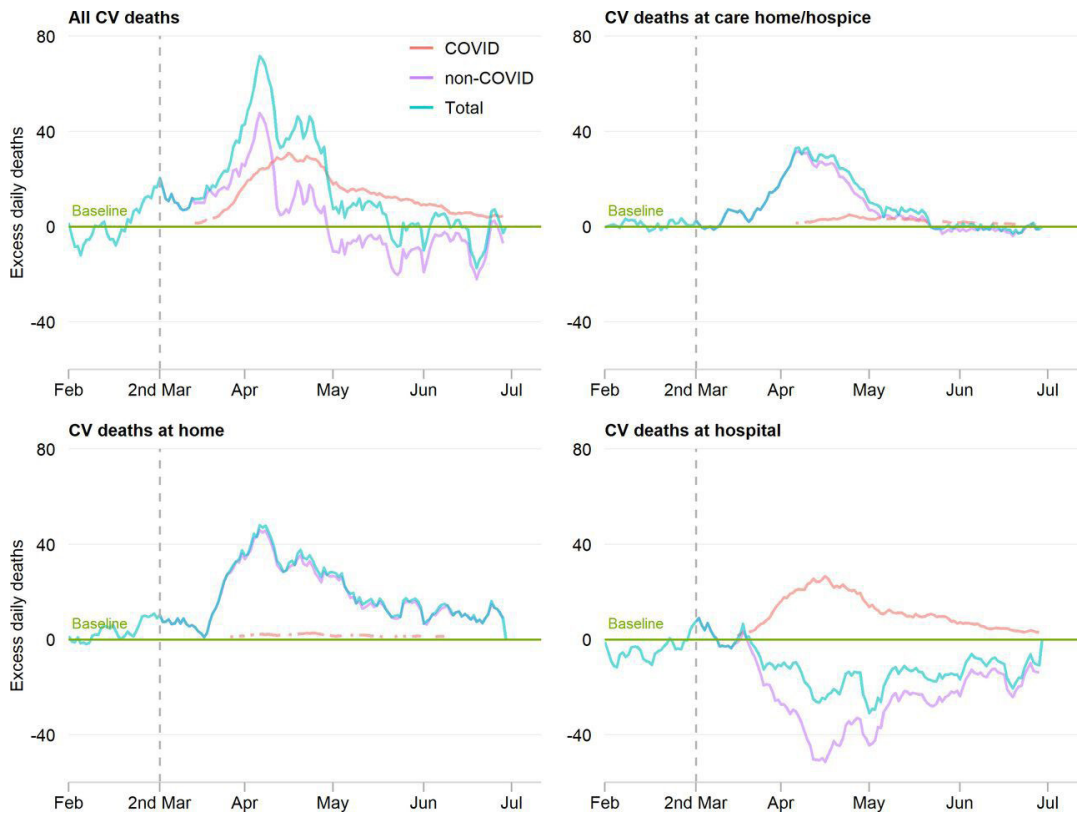

Figure 1 Time series of acute cardiovascular (CV) deaths, by place of death. The number of daily CV deaths is presented using a 7-day simple moving average (indicating the mean number of daily CV deaths for that day and the preceding 6 days) from 1 February 2020 up to and including 30 June 2020, adjusted for seasonality. The number of non-COVID-19 excess CV deaths each day from 1 February 2020 were subtracted from the expected daily death estimated using Farrington surveillance algorithm in the same time period. The green line is a zero historical baseline. The red line represents daily COVID-19 CV death from 2 March to 30 June 2020; the purple line represents excess daily non-COVID-19 CV death from 2 March to 30 June 2020 and the blue line represents the total excess daily CV death from 1 February to 30 June 2020.

with a markedly lower risk of in-hospital mortality (OR 0.25, p<0.001) and improved long-term outcomes (figure 2 ).

'We could (and should) do better' in preventing and treating infective endocarditis plead Scully et al. ${ }^{4}$ They conclude that: 'As the present data from South Korea demonstrate, IE remains associated with poor outcomes and its incidence is increasing in many countries around the world. Greater public health awareness is warranted alongside renewed emphasis on education of patients at risk (with particular regard to prompt symptom reporting and maintenance of good oral and cutaneous hygiene), early diagnosis, timely referral and specialist care. Once suspected or diagnosed, early involvement of a dedicated Endocarditis Team is essential in managing these patients combined with early, appropriate antibiotic therapy and decisions regarding the need for surgery and its timing.'

Another interesting paper in this issue of Heart by Onishi and colleagues ${ }^{5}$ describes the diagnosis and outcomes of triglyceride deposit cardiomyovasculopathy (TGCV) which is seen in about $20 \%$ of haemodialysis patients with suspected coronary artery disease. At median follow-up of 4.7 years, the composite primary endpoint of CVD death, nonfatal myocardial infarction and non-fatal stroke occurred in $52.3 \%$ of the definite TGCV patients compared with $27.3 \%$ in those with probable TGCV and $9.1 \%$ of the non-TGCV patients. In the accompanying editorial, Nakajima ${ }^{6}$ explains the causes of TGCV and discusses the diagnostic approach. In brief, 'The principal disorder in TGCV is defective intracellular lipolysis, which causes excessive triglyceride accumulation in 


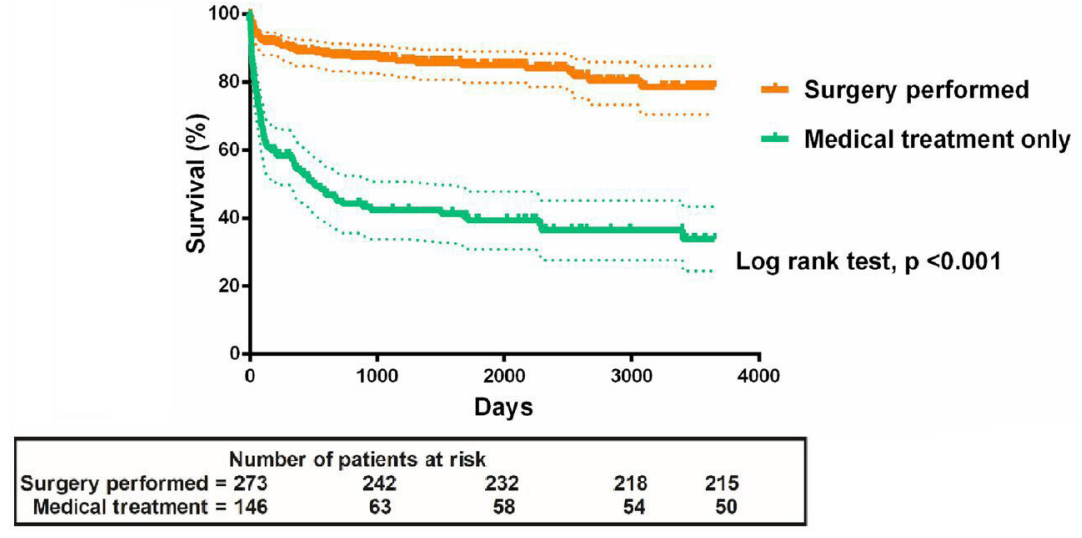

Figure 2 Kaplan-Meier curves of the long-term survival rates of patients with infective endocarditis who underwent surgery versus those who underwent medical treatment only.

the myocardium and coronary artery vascular smooth muscle cells, leading to heart failure and coronary artery disease with a poor prognosis.' Diagnosis is based on the presence of impaired long-chain fatty acid metabolism or triglyceride deposition in the myocardium in combination with clinical major and minor criteria and supportive items.

The Education in Heart article in this issue $^{7}$ reviews the prevalence and predictors of neurocognitive and psychosocial impairment among adults with congenital heart disease followed by a discussion of how these issues can be mitigated over the patient's lifespan.

Readers will also want look at the review article $^{8}$ on the emerging mechanistic models that link atrial fibrosis, atrial fibrillation and stroke given the implications of these models for new approaches to prevention of adverse clinical events (figure 3). Boyle et al outline 'a vision of a future paradigm integrating simulations in formulating personalised treatment plans for each patient.'

Funding The authors have not declared a specific grant for this research from any funding agency in the public, commercial or not-for-profit sectors.

Competing interests None declared.

Patient and public involvement Patients and/or the public were not involved in the design, or conduct, or reporting, or dissemination plans of this research.

Patient consent for publication Not required.

Provenance and peer review Commissioned; internally peer reviewed.

This article is made freely available for use in accordance with BMJ's website terms and conditions for the duration of the covid-19 pandemic or until otherwise determined by BMJ. You may use, download and print the article for any lawful, non-commercial purpose (including text and data mining) provided that all copyright notices and trade marks are retained.

(c) Author(s) (or their employer(s)) 2021. No commercia re-use. See rights and permissions. Published by BMJ.

$$
\text { A Check for updates }
$$

To cite Otto CM. Heart 2021;107:89-90.

Heart 2021;107:89-90.

doi:10.1136/heartjnl-2020-318759

ORCID iD

Catherine M Otto http://orcid.org/0000-0002-05279392

\section{REFERENCES}

1 Wu J, Mamas MA, Mohamed MO, et al. Place and causes of acute cardiovascular mortality during the COVID-19 pandemic. Heart 2021;107:113-9.

2 Singh T, Newby DE. Is the fear of disease worse than the disease itself? Heart 2021:107:91-2.

$3 \mathrm{Kim} \mathrm{JH}$, Lee HJ, Ku NS, et al. Infective endocarditis at a tertiary care hospital in South Korea. Heart 2021;107:135-41.

4 Scully PR, Woldman S, Prendergast BD. Infective endocarditis: we could (and should) do better. Heart 2021:107:96-8

5 Onishi T, Nakano Y, Hirano K-l, et al. Prevalence and clinical outcomes of triglyceride deposit cardiomyovasculopathy among haemodialysis patients. Heart 2021;107:127-34.

6 Nakajima K. Triglyceride deposit cardiomyovasculopathy: how to recognise a new disease entity. Heart 2021; 107:93-5.

7 Kovacs $\mathrm{AH}$, Bellinger DC. Neurocognitive and psychosocial outcomes in adult congenital heart disease: a lifespan approach. Heart 2021;107:159-67.

8 Boyle PM, del Álamo JC, Akoum N. Fibrosis, atrial fibrillation and stroke: clinical updates and emerging mechanistic models. Heart 2021;107:99-105. 